\title{
Heat transfer studies for a crystal in a synchrotron radiation beamline
}

\author{
A K SINHA \\ Synchrotron Utilisation and Materials Research Division, Raja Ramanna Center \\ for Advanced Technology, Indore 452013 \\ e-mail: anil@ rrcat.gov.in
}

MS received 14 August 2007; revised 11 November 2008

\begin{abstract}
Heat load studies have been performed for the first crystal of a double crystal monochromator to be installed in a beamline of the $2.5 \mathrm{GeV}$ synchrotron radiation source Indus-2. Finite element analysis (FEA) has been used to calculate the temperature distribution and the mechanical distortions on these crystals. Several cases of cooling schemes and heat loads have been studied. Based on these FEA results, the analytical relationships available in the literature have been modified. It is shown that modified analytical results compare well with the empirical results obtained from FEA. The optimisation of the cooling conditions can be achieved by doing FEA calculations for only one case. All other cases can then be calculated by using analytical relations proposed here. The proposed analytical equations are generic in nature and can be applied for any source-crystal combination and therefore would be useful for performance prediction of any new monochromator on a new synchrotron source without taking recourse to time consuming, computation-intensive FEA.
\end{abstract}

Keywords. Synchrotron radiation; heat load; finite element analysis; silicon crystal.

\section{Introduction}

The $2.5 \mathrm{GeV}, 300 \mathrm{~mA}$ Indian synchrotron radiation (SR) source Indus-2 (Raja Rao 1998) is operational at Raja Ramanna Center for Advanced Technology, Indore. Some initial experiments using SR is under way. Indus 2 has been designed to emit continuous SR in the hard X-ray regime from its bending magnets, as well as the insertion devices (wavelength shifter, wigglers) installed in the straight sections of the storage ring. The high flux and high brightness of the synchrotron source can be utilised if the high brightness is transferred to the experimental station with minimal loss in the brightness. One source of degradation is thermo-mechanical distortion in the optical elements because of the large heat load. For instance, the performance of a crystal monochromator installed in the beamline degrades because of the distortion induced by thermal irradiation by synchrotron radiation. To minimize this degradation, it is customary to cool the crystal. A number of 
Table 1. Indus-2 synchrotron radiation source characteristics.

\begin{tabular}{lll}
\hline & \multicolumn{2}{c}{ Indus-2 } \\
\hline Electron energy & $2.5 \mathrm{GeV}$ & \\
Max. electron current & $300 \mathrm{~mA}$ & Wavelength shifter \\
& Bending magnet & $5 \cdot 0 \mathrm{~T}$ \\
Magnetic field & $1.5 \mathrm{~T}$ & $0.6 \AA$ \\
Critical wavelength & $2.0 \AA$ & $90 \mathrm{~W}$ \\
Power radiated* & $28 \mathrm{~W}$ & \\
\hline
\end{tabular}

* per mrad of horizontal acceptance

cooling schemes are reported in literature. For intermediate heat loads, an indirect cooling scheme is followed (Jensen et al 1995, Michaud 1986), in which crystals are kept on a water-cooled copper base. The scheme often uses some material between base plate and crystal to improve the thermal contact. A direct cooling scheme, by making channels in the crystal, is adopted for high heat loads (Mochizuki et al 1995, Oversluizen et al 1989, Arthur et al 1992). There are variations to these cooling schemes. For ultra high heat loads (radiation from insertion devices) cooling is direct and the coolant is liquid gallium (Smither et al 1989). In addition, some improvised methods like use of thin crystals (Freund et al 1992), Si crystals at cryogenic temperatures (Knapp et al 1995, Kuzay 1992, Wang et al 1995), jet cooling (Berman \& Hart 1992) etc. are also used. Finite element (FE) analysis is done for optimisation of the cooling geometry as well as the cooling parameters for individual beamlines. In addition, the mechanical distortion results are used in the raytracing program to estimate the realistic performance of a beamline. In spite of numerous FE calculations, very little guideline exists for performance prediction of a new monochromator on a new synchrotron source. Analytical formulae (Smither et al 1989), based on simple physical arguments, have limited applicability in predicting the performance of crystals.

In this paper, analytical relations have been worked out for maximum temperature rise in the crystal as a function of Bragg angle and these are compared with the empirical relations obtained from rigorous FE calculations using finite element software Ansys (Ansys 1998). Also various components of the crystal distortion have been calculated using analytical relations and empirical results obtained using FE calculations. Two cooling geometries, viz. the indirect cooling and the direct cooling of the crystals are studied here.

\section{Calculation details}

Indus- 2 characteristics are given in table 1 . The total power radiated by the wavelength shifter is $90 \mathrm{~W}$ for $1 \mathrm{mrad}$ of horizontal acceptance, which is large enough to necessitate convection cooling of the first optical elements of the beamline. In the beamlines proposed on Indus-2, crystal monochromator can be either the first optical element (OE), immediately after the frontend, or it can be the second OE, preceded by a mirror. Both these cases have been considered for FE calculations. In the second case the absorbed heat load is about 3.5 times less than the first case because of the absorption in the preceding mirror. In addition for each location of the crystal, two convection film coefficients have been taken for FE calculations. Also, two cooling geometries have been considered. These are indirect (figure 1a) and direct 

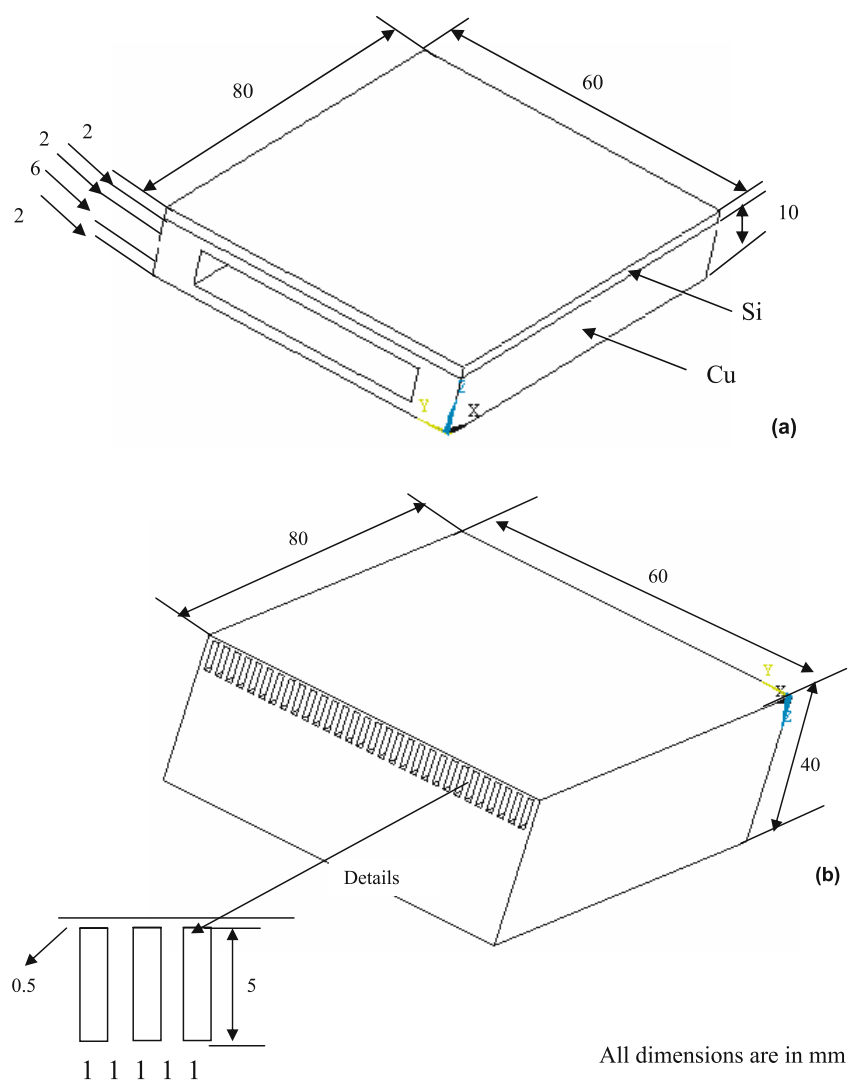

Figure 1. Cooling geometries of indirect cooled crystal (figure 1a) and direct cooled crystal (figure 1b). The dimensions of the crystals are $80 \mathrm{~mm} \times 60 \mathrm{~mm} \times$ $2 \mathrm{~mm}$ (figure 1a) and $80 \mathrm{~mm} \times$ $60 \mathrm{~mm} \times 40 \mathrm{~mm}$ (figure $1 \mathrm{~b}$ ). For the case of direct cooling, the dimensions of the cooling channels are $5 \mathrm{~mm} \times 1 \mathrm{~mm}$ and the separation between the channels is $1 \mathrm{~mm}$. The channels are made $0.5 \mathrm{~mm}$ below the surface of the crystal. There are 30 channels in all.

(figure 1b) cooling. Table 2 gives the parameters for indirect cooling for the various cases considered. All the symbols used in this paper are defined in 'List of symbols' at the end (before references).

Table 2. Various cases and corresponding input parameters for indirect cooling of the crystal. Distance of the crystal from the source point has been taken to be $22 \mathrm{~m}$.

\begin{tabular}{llccc}
\hline Case & Crystal position & $\begin{array}{c}h_{f} \\
{\left[\mathrm{~W} / \mathrm{cm}^{2} /{ }^{\circ} \mathrm{C}\right]}\end{array}$ & $\begin{array}{c}\text { Power absorbed* } \\
{[\mathrm{W}]}\end{array}$ & $\begin{array}{c}\text { Power density } \\
{\left[\mathrm{W} / \mathrm{mm}^{2}\right]}\end{array}$ \\
\hline Case I & $1^{\text {st }}$ optical element & 0.45 & 81 & $0 \cdot 6$ \\
Case II & $1^{\text {st }}$ optical element & 0.60 & 81 & $0 \cdot 6$ \\
Case III & $2^{\text {nd }}$ Optical Element & 0.45 & $22 \cdot 5$ & $0 \cdot 17$ \\
Case IV & $2^{\text {nd }}$ Optical Element & 0.60 & $22 \cdot 5$ & $0 \cdot 17$ \\
\hline
\end{tabular}

* For $1 \mathrm{mrad}$ of horizontal acceptance

$\S_{\text {crystal normal to the incident radiation }}$ 


\subsection{Finite element analysis}

The radiation emitted by a synchrotron source is anisotropic and moreover the cooling geometry of the crystal is also generally complicated. Therefore, it is difficult to estimate the temperature distribution, and hence the mechanical distortion, analytically. Finite element (FE) analysis is commonly used to study the problem. In this paper, we have done FE calculations for two cooling geometries for obtaining temperature distribution in the crystal using FE software Ansys. Figure 1 shows the two cooling geometries of the crystal along with dimensions. The crystals are cooled by flowing water at $25^{\circ} \mathrm{C}(\mathrm{RT})$. For indirect (figure 1a) cooling, a $2 \mathrm{~mm}$ thick $\mathrm{Si}(111)$ crystal is kept on water cooled copper base. To improve thermal contact, In-Ga alloy paste is painted in between $\mathrm{Si}$ and copper surfaces. For direct cooling, on the other hand, 30 rectangular channels are made in the crystal itself. Cooling fluid (water) is flown through these channels. The channel dimensions are similar to that in the paper by Assoufid et al (1995).

The flux of cooling fluid in the case of direct cooling is approximately $141 / \mathrm{m}$ that is equivalent to a fluid velocity of $100 \mathrm{~cm} / \mathrm{s}$, which is within the streamline range. The average film coefficient is calculated using Baker and Tessier equation (Rohsenow \& Choi 1961):

$$
h_{f}=\frac{A_{1} K}{d}+\frac{A_{2} k^{0 \cdot 6} C_{v}^{0 \cdot 4} V_{a}^{0 \cdot 8}}{d^{0 \cdot 2} v^{0 \cdot 8}}
$$

and found to be $0.6 \mathrm{~W} / \mathrm{cm}^{2} /{ }^{\circ} \mathrm{C}$. Constants $A_{1}$ and $A_{2}$ are empirically determined to be approximately 1 . Turbulent flow gives more effective cooling but the mechanical vibrations degrade the monochromator performance. For comparison and to evaluate the cooling dependence on water flow rate, we have done FE calculations for $h_{f}=1.2 \mathrm{~W} / \mathrm{cm}^{2} /{ }^{\circ} \mathrm{C}$ as well, which also falls in the streamline flow region. Similarly for direct cooling, flow rate of $\sim 101 / \mathrm{m}$ gives a film coefficient of $1.2 \mathrm{~W} / \mathrm{cm}^{2} /{ }^{\circ} \mathrm{C}$. We have also done calculations for $h_{f}=0.6 \mathrm{~W} / \mathrm{cm}^{2} /{ }^{\circ} \mathrm{C}$ for the same geometry. This means decreasing the velocity by approximately 3 times compared to the case of $h_{f}=1.2 \mathrm{~W} / \mathrm{cm}^{2} /{ }^{\circ} \mathrm{C}$.

Heat load is approximately Gaussian in the vertical plane and uniform in the horizontal plane. Temperature distribution has been calculated using thermal element solid 87 in ANSYS 5.7. The elements have 10 nodes and are tetragonal in shape. These elements are recommended for the case that is to be transformed to structural problem. The thermal strain has been calculated by transforming thermal element to structural element (element solid 92). Since the temperature rises are small, the physical properties of $\mathrm{Si}$ and $\mathrm{Cu}$ were taken to be constant (RT value), independent of temperature.

\section{Results and discussions}

Using FE analysis, we have estimated the temperature distribution and distortion in the first crystal of a double crystal monochromator (DCM) for indirect as well as direct cooling of the crystal, for the four cases given in table 2. Various Bragg angle settings of the crystal corresponding to photon energies of 5 to $25 \mathrm{keV}$ have been considered. Section 3.1 gives the temperature distribution results for indirect cooling. In this section, we first obtain temperature distribution in the crystal using rigorous FE analysis. It is seen that there is a large discrepancy between the values of maximum temperature rise in the crystal calculated analytically using existing formulae and the FEM results (Smither et al 1989). We, considering some realistic assumptions, have modified analytical formulae. The results based on these analytical formulae are found to be matching quite well with the empirical relations obtained by fitting FEM 

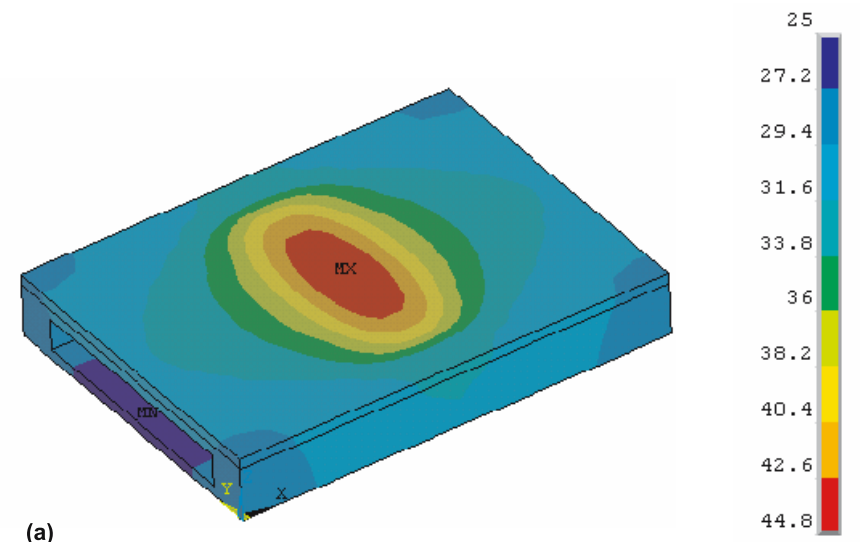

Temperature distribution in ${ }^{\circ} \mathrm{C}$

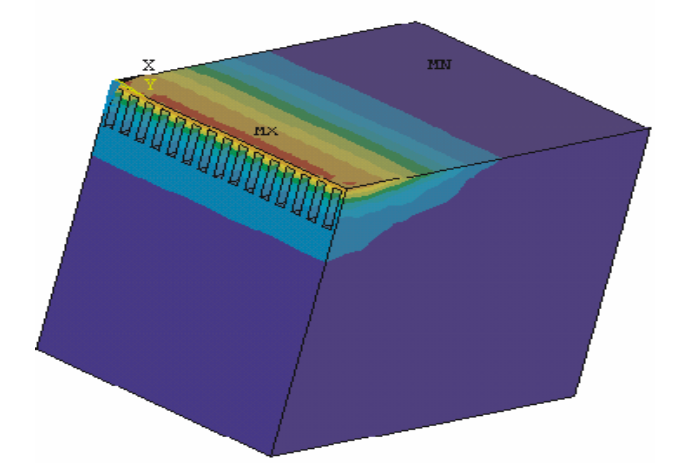

(b)

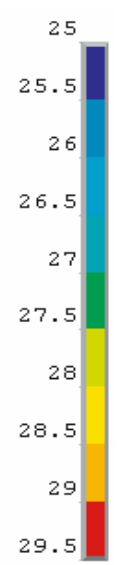

Figure 2. Typical temperature distribution on the crystal as obtained from the finite element analysis for indirect (figure 2a) and direct (figure 2b) cooled crystals. Shown contours are for $81 \mathrm{~W}$ (for $1 \mathrm{mrad}$ horizontal acceptance) and Bragg angle of $23.3^{\circ}$. The film coefficient values are $0.6 \mathrm{~W} / \mathrm{cm}^{2} /{ }^{\circ} \mathrm{C}$ for indirect cooled crystals and $0.6 \mathrm{~W} / \mathrm{cm}^{2} /{ }^{\circ} \mathrm{C}$ for direct cooled crystals. Figure $2 \mathrm{a}$ shows the full crystal, whereas, figure $2 \mathrm{~b}$ shows half of the crystal.

data. In section 3.2, temperature results for direct cooling are similarly derived and discussed. The crystal distortion results have been obtained by FEM as well as analytically in section $3 \cdot 3$.

\subsection{Temperature results for indirect cooling}

For indirect cooling, figure 2 a shows a representative temperature distribution as obtained by $\mathrm{FE}$ analysis. This contour plot is for crystal as the first optical element (absorbed power $=$ $81 \mathrm{~W}$ for $1 \mathrm{mrad}$ of horizontal acceptance). The Bragg angle is $23.3^{\circ}$ and $h_{f}=0.6 \mathrm{~W} / \mathrm{cm}^{2} /{ }^{\circ} \mathrm{C}$. The figure shows that the maximum temperature reached on the crystal surface is $44.8^{\circ} \mathrm{C}$. Similar calculations have been done for all Bragg angles. Figure 3a shows $\Delta T_{\max }-\Delta T_{3 f}$ plotted as a function of photon energy reflected by the crystal, for the four cases given in 


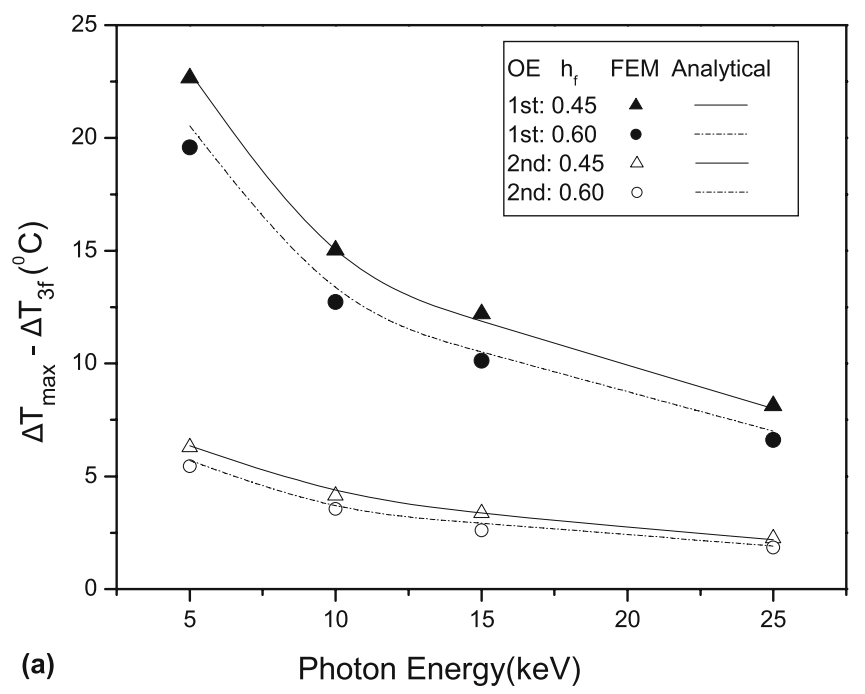

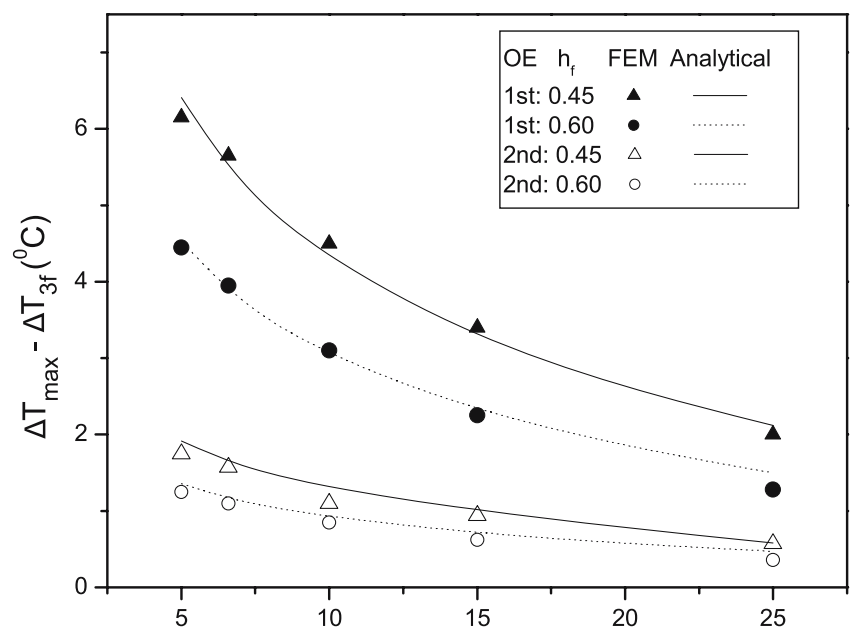

(b)
Figure 3. Difference $\left(\Delta T_{\max }-\right.$ $\left.\Delta T_{3 f}=T_{1}-T_{3}\right)$ between maximum temperature on the surface of the crystal $\left(T_{1}\right)$ and average temperature of the out flowing water $\left(T_{3}\right)$ is plotted as a function of photon energy, which is being reflected by the crystal, for the case of indirect cooling (figure 3a) and direct cooling (figure $3 b$ ). Scattered points are the results of finite element analysis and the curves are empirical fit to the data using Eq. (2a) for figure $3 a$ and Eq. (8) for figure $3 b$. Various curves are for different values of power absorbed by the crystal and the convection film coefficients.

table 1. The scattered points are the FE results and the lines show empirical fit to them using the relation:

$$
\Delta T_{\max }-\Delta T_{3 f}=K_{1} P_{\max }+\frac{K_{2} P_{\max }^{1 / 2} P_{a b s}^{1 / 2}}{h_{f}^{1 / 2}} .
$$

Where $K_{1}$ and $K_{2}$ are the fitting constants. Values of the constants found from the empirical fit are 0.22 and 0.27 respectively. We find from the figure that the fits are quite good. Next, we will compare these results with the analytical results.

For discussion, we first take the case of the crystal being the first $\mathrm{OE}, h_{f}=0.45 \mathrm{~W} / \mathrm{cm}^{2} /{ }^{\circ} \mathrm{C}$ and Bragg angle of $23.3^{\circ}$ (set to reflect $5 \mathrm{keV}$ photons). The $\mathrm{FE}$ calculation for this case gives maximum temperature increase $\left(\Delta T_{\max }\right)$ on the surface of the crystal $\sim 23^{\circ} \mathrm{C}$ (see figure $2 \mathrm{a}$ ). Analytically, $\Delta T_{\max }$ can be written as sum of temperatures of various parts of the crystal as 
below

$$
\Delta T_{\max }=\Delta T_{12}+\Delta T_{23}+\Delta T_{3 f}
$$

Using simple physical arguments, various components in Eq. (2b) are given as Ref. (Smither 1989)

$$
\begin{aligned}
\Delta T_{12} & =\frac{D P_{\max }}{K} \\
\Delta T_{23} & =\frac{P_{\max }}{h_{f}} \\
\Delta T_{3 f} & =\frac{P_{a b s} N}{m C_{v}} .
\end{aligned}
$$

Calculating the temperature values using the above equations, we get $\Delta T_{12}=2.9^{\circ} \mathrm{C}$, $\Delta T_{3 f}=0.5^{\circ} \mathrm{C}$ and $\Delta T_{23}=32^{\circ} \mathrm{C}$. So, $\Delta T_{23}$ obtained using the analytical relation $\left(32^{\circ} \mathrm{C}\right)$ is much larger than that obtained using FEM $\left(\sim 20^{\circ} \mathrm{C}\right.$, see figure $\left.2 \mathrm{a}\right)$. This discrepancy comes from the fact that while Eq. $3 \mathrm{~b}$ assumes that $T_{2}$ is the temperature throughout the cooling channel cross section whereas in reality there is a large variation in the temperature across the cross section. Equation $3 \mathrm{~b}$ in the same form can be used if $h_{f}$ is replaced by an effective film coefficient, $h_{w}$. To calculate $h_{w}$ we assume that the lowest temperature of the copper block is $T_{f}$. The temperature of the copper water interface decreases linearly from $T_{2}$ to $T_{3}$ for the top plate of the cooling channel and from $T_{3}$ to $T_{f}$ for the bottom plate of the cooling channel. Therefore, total heat taken out by the four walls of the copper cooling channel is $\left(h_{f} / 2\right)\left\{A_{1} \cdot \Delta T_{23}+A_{1} \cdot \Delta T_{3 f}+2 A_{2} \cdot\left(\Delta T_{23}+\Delta T_{3 f}\right) / 2\right\}$. Since the average temperature of the cooling liquid is $T_{3}$, the total heat convection in terms of the effective film coefficient is $h_{w} \cdot 2\left(A_{1}+A_{2}\right) \cdot \Delta T_{3 f}$. Equating the two, we get

$$
h_{w}=\frac{\left(h_{f} / 2\right)\left\{A_{1} \Delta T_{23}+A_{1} \Delta T_{3 f}+2 A_{2}\left(\Delta T_{23}+\Delta T_{3 f}\right) / 2\right\}}{2\left(A_{1}+A_{2}\right) \Delta T_{3 f}} .
$$

Since $T_{3 f} \ll T_{23}$, Eq. 4 reduces to

$$
h_{w}=\frac{h_{f} \Delta T_{23}}{4 \Delta T_{3 f}} .
$$

Replacing $h_{f}$ in Eq. 3 by $h_{w}$, we get

$$
\Delta T_{23}=\frac{\left(4 N / m C_{v}\right)^{1 / 2} P_{\max }^{1 / 2} P_{a b s}^{1 / 2}}{h_{f}^{1 / 2}} .
$$

From Eqs. 2, 3 and 6, we get

$$
\Delta T_{\max }-\Delta T_{3 f}=K_{1} P_{\max }+\frac{K_{2} P_{\max }^{1 / 2} P_{a b s}^{1 / 2}}{h_{f}^{1 / 2}}
$$

where $K_{1}=D / K_{\text {eff }}=0.2$ and $K_{2}=\left(4 \mathrm{~N} / \mathrm{mC}_{\mathrm{v}}\right)^{1 / 2}=0.16$ for the case under discussion. It is seen that the above equation is the same as Eq. 2a, obtained from empirical fitting of FEM results. The values of constants for the empirical fits were $K_{1}=0.22$ and $K_{2}=0 \cdot 27$. 
It is seen that the functional dependence have been correctly predicted by the analytical calculations. In addition, there is an excellent agreement between $K_{1}$ obtained empirically from FEM (0.22) and that obtained analytically (0.2). However, the two values of $K_{2}$ are off by a factor of $\sim 1 \cdot 5$. This is not unexpected as only approximate temperature values were taken in the derivation of Eq. (5). The discrepancy also indicates that it is not straightforward to estimate the value of the constant $K_{2}$, without going into details of the cooling geometry and the heat loading. However, the constants $K_{1}$ and $K_{2}$ need to be obtained only once for a particular geometry and these values then give good estimates for different heat loads and different $h_{f}$. This shows that the constants are independent of heat load levels and value of $h_{f}$. Therefore, the optimisation of the cooling geometry as well as convection film coefficient etc. can be done by performing FEM calculations for one value of these parameters and the values for other cases can then be derived using Eq. 2a. Thus, the functional dependence of the temperature distribution on input parameters can be correctly predicted analytically using the modified formula.

\subsection{Temperature results for direct cooling}

Temperature distribution, derived from FE calculations, for a directly cooled crystal is given in figure $2 \mathrm{~b}$. This plot is for absorbed power of $81 \mathrm{~W}$ (for $1 \mathrm{mrad}$ of horizontal acceptance), Bragg angle of $23.3^{\circ}$ and $h_{f}=0.45 \mathrm{~W} / \mathrm{cm}^{2} /{ }^{\circ} \mathrm{C}$. Similar FE calculations have been done for other cases. Figure $3 \mathrm{~b}$ shows $\left(\Delta T_{\max }-\Delta T_{3 f}\right)$ plotted as a function of photon energy for cooling geometry shown in figure $1 \mathrm{~b}$. FE results are shown by the scattered points along with the fit to the point using the empirical equation:

$$
\Delta T_{\max }-\Delta T_{3 f}=\frac{C_{1} P_{\max }}{h_{f}^{1 / 2}}+\frac{C_{2} P_{\max }^{1 / 2} P_{a b s}^{1 / 2}}{h_{f}^{1 / 2}} .
$$

The values of $C_{1}$ and $C_{2}$ are found to be $0 \cdot 3$ and $0 \cdot 11$ respectively. Again the fit between the FEM calculations and the empirical data are good for all cases of heat load, film coefficient and Bragg angle. This shows the validity of functional dependence of Eq. 8. In this geometry it is not straightforward to calculate effective $\mathrm{D}$ and $\mathrm{K}$ analytically because there is heat flow along both the horizontal and the vertical directions. However, the only difference between the functional dependence for this case and the case of indirect cooling is the dependence of the two terms on $h_{f}^{1 / 2}$. Dependence of the first term on $h_{f}$ is explained by arguing that larger the $h_{f}$ larger will be the heat flow along horizontal direction (on x-y plane); and effective D will be smaller giving smaller $\Delta T_{12}$. This explains why $\Delta T_{12}$ depends inversely upon $h_{f}$. Similarly, $\Delta T_{23}$ will have a slower dependence on $h_{f}$. This is also observed in the empirical relation (Eq. 8).

\subsection{Crystal distortion results}

The three components of the crystal distortion (Lenardi 1993) are bowing, bump and increase in Darwin width because of the change in d value of the crystal. These distortions are caused due to the non-uniform temperature distribution in the crystal. Bowing (bending) is caused by the thermal expansion of the crystal in the direction parallel to the surface; due to the thermal variation along the depth of the crystal. Bump, on the other hand, is caused by the expansion of the crystal in the direction perpendicular to the surface due to the thermal variation along the direction parallel to crystal surface. For Indus-2 heat load, the angular errors due to first two effects are comparable and the third effect is negligible. Therefore, we consider only the first two components. 


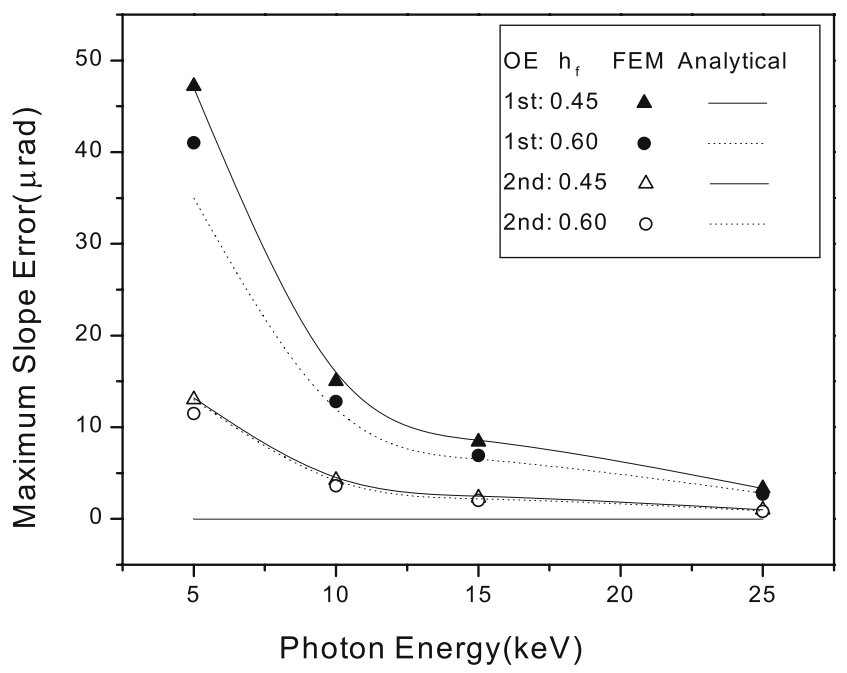

Figure 4. Maximum thermal slope error on the crystal calculated using finite element structural analysis (scattered points) and using analytical relations (continuous curves), for the case of indirect cooling. Various curves are for different values of power absorbed by the crystal and the convection film coefficients.

3.3a Bump: The Bump height $(\mathrm{H})$ is calculated using the following equation (Lenardi 1993):

$$
H=\alpha_{\text {eff }} D_{\text {eff }}\left(\Delta T_{12} / 2+\Delta T_{23}+\Delta T_{3 f}\right) .
$$

And, the maximum slope error is $\theta_{\max }(\mathrm{rad})= \pm 1.43 \cdot \mathrm{H} / \mathrm{FWHM}$, where FWHM is the full width at half maximum of the Gaussian heat load. $\alpha_{\text {eff }} D_{\text {eff }}$ in the case of indirect cooling is calculated using the equations:

$$
\begin{aligned}
& \alpha_{S i} D_{S i} \Delta T_{1 I}+\alpha_{C u} D_{C u} \Delta T_{I 2}=\alpha_{\text {eff }} D_{\text {eff }} \Delta T_{12} \\
& \Delta T_{1 I}+\Delta T_{I 2}=\Delta T_{12}
\end{aligned}
$$

and

$$
\frac{K_{S i} \Delta T_{1 I}}{D_{S i}}+\frac{K_{C u} \Delta T_{I 2}}{D_{C u}}=\frac{K_{\mathrm{eff}} \Delta T_{12}}{D_{\mathrm{eff}}}
$$

and found to be $1.13 \times 10^{-6} \mathrm{~cm} /{ }^{\circ} \mathrm{C}$. Here $\Delta T_{1 I}$ is the temperature difference between the surface of the crystal and $\mathrm{Si}-\mathrm{Cu}$ interface. Similarly $\Delta T_{I 2}$ is the temperature difference between the interface and the bottom of $\mathrm{Cu}$. Above equations represent respectively the thermal strain and conduction equations across the interface. Since, everything in Eq. 9 is known, we can estimate $\mathrm{H}$, and hence $\theta_{\max }$, analytically. Also, to see the correctness of this analytically derived bump height, it has also been calculated from rigorous FE structural analysis. FEM results for $\theta_{\max }$ are plotted in figure 4 (scattered points) along with $\theta_{\max }$ obtained analytically (continuous line) for the four cases of heat absorption and cooling conditions (table 2). The figure shows that the agreement between the two is good. This shows that for indirect cooling geometry, crystal deformation can be estimated analytically if temperature values are known. Figure 4 shows that the maximum slope error is $\sim 47 \mu \mathrm{rad}$ for $P_{a b s}=81 \mathrm{~W} / \mathrm{mrad}$ of horizontal acceptance and $h_{f}=0.45 \mathrm{~W} / \mathrm{cm}^{2} /{ }^{\circ} \mathrm{C}$, when the crystal is set to reflect $5 \mathrm{keV}$ photons (Bragg angle $\left.=23 \cdot 3^{\circ}\right)$. This slope error is less than the Darwin Width $(60 \mu \mathrm{rad})$ of Si $(111)$ for $5 \mathrm{keV}$ photons. Therefore the cooling design is acceptable, even for maximum heat load and lower cooling. 


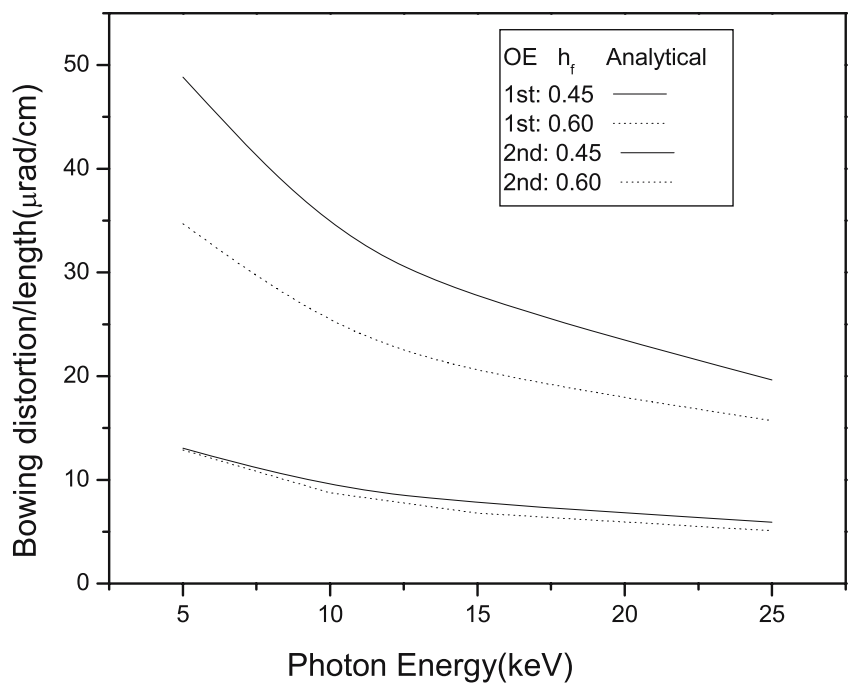

Figure 5. Maximum bowing distortion per unit length calculated using analytical relations (continuous curves), for the case of indirect cooling. Various curves are for different values of power absorbed by the crystal and the convection film coefficients.

In the case of direct cooling, Eq. 9 is used to calculate the bump. In this case, taking $D_{\text {eff }}$ to be the thickness above the cooling channel $(0.05 \mathrm{~cm})$ of the crystal does not provide a good fit. We have taken the value of $D_{\text {eff }}$ to be $0.55 \mathrm{~cm}$, which is the distance from the surface of the crystal to the bottom of cooling channel to get a good fit. Maximum slope error calculated analytically is compared with those obtained from structural FE analysis (figure 4). The agreement between the two is found to be reasonable. Maximum slope error obtained using FEM for the worst case of heat load and lower value of film coefficient is less than $15 \mu \mathrm{rad}$, which is well within acceptable limits.

3.3b Bowing: Bowing Radius $(R)=D /\left(\alpha \Delta T_{12}\right)$ (Lenardi 1993) and bowing distortion per unit length $\Delta \theta_{b}=1 / R$ radian/cm have been calculated by estimating $\Delta T_{12}$ using the empirical relations $\Delta T_{12}=T_{\max }-T_{3}-0 \cdot 27 \cdot\left(P_{\max } P_{a b s} / h_{f}^{1 / 2}\right.$ for indirect cooling and $\Delta T_{12}=C_{1} \cdot P_{\max } / h_{f}^{1 / 2}$ for direct cooling. In figure $5, \Delta \theta_{b}(\mu \mathrm{rad} / \mathrm{cm})$ as a function of photon energy has been plotted for the four cases (table 2) for indirect cooling. From the figure we find that the maximum bowing is $\sim 50 \mu \mathrm{rad} / \mathrm{cm}$. For the case of direct cooling, the maximum bowing is $\sim 20 \mu \mathrm{rad} / \mathrm{cm}$, which is much less than the case of indirect cooling. These results are expected and in agreement with the literature (Michaud 1986, Oversluizen et al 1989, Assoufid et al 1995).

We find that, in general, there is a good agreement between the temperature as well as crystal deformation results calculated using thermal and structural FE analysis respectively with the analytical formulae. In the case of indirect cooling, which is rather simple cooling geometry, we are able to explain all the components of the empirical temperature rise formula satisfactorily. Also in this case, the crystal deformation results are well accounted for. However, in the case of direct cooling, the geometry of cooling is complicated and hence cooling phenomena is complex. So some of the terms in the empirical temperature rise formula is only partially explained. However, the fit between FEM values and the values obtained using empirical formula for various values of $P_{\max }$ and $h_{f}$ is satisfactory. Also, for explaining crystal distortion results, we have taken the value of $D_{\text {eff }}$ rather arbitrarily to be the distance between the surface of the crystal and bottom of the cooling channel, which needs explana- 
tion. Therefore, more theoretical work is required to be done to explain empirical results in the case of direct cooling of crystals.

\section{Conclusions}

For wavelength shifter insertion device source on Indus- 2 synchrotron ring ( $2.5 \mathrm{GeV}, 300 \mathrm{~mA})$, temperature distribution and thermal distortion have been studied by Finite Element Analysis; for the first crystal of a double crystal monochromator. Calculations have been done for two cases namely one in which the crystal is the $1^{\text {st }}$ optical element (OE) and second in which crystal is the $2^{\text {nd }} \mathrm{OE}$, preceded by a mirror. In the first case when the crystal is the first $\mathrm{OE}$, the total incident heat load is $81 \mathrm{~W}$ per mrad of horizontal acceptance, whereas when the crystal is second optical element the incident heat load is $\sim 22.5 \mathrm{~W}$ for $1 \mathrm{mrad}$ of horizontal acceptance. Also, two geometries of cooling have been discussed. In indirect cooling, thin crystal $(2 \mathrm{~mm})$ is kept on a water-cooled Cu plate. In direct cooling, rectangular channels are drilled in the crystal and water flows through them. As expected, the temperature rise is the maximum for the case when the crystal is the first $\mathrm{OE}$ and is set to reflect the lowest energy photons $(5 \mathrm{keV})$. The temperature rise is $\sim 23^{\circ} \mathrm{C}$. Corresponding slope error as well as bowing distortion have been calculated and found to be $47 \mu \mathrm{rad}$ and $50 \mu \mathrm{rad} / \mathrm{cm}$ respectively. These are within $100 \%$ of the Darwin width (Assoufid et al 1995). This shows that indirect cooling, which is less efficient but easier to achieve is acceptable for Indus-2 heat load, even if the crystal is the first optical component.

Empirical relations for calculation of maximum temperature rise in the crystal have been worked out from FEM results for both cooling geometries. Existing analytical formulae have been modified. It is shown that the functional dependences of the maximum temperature rise on absorbed power; the modified analytical relations proposed by us correctly predict convection film coefficient and Bragg angle. This is very handy, because the optimisation of the cooling conditions can be achieved by doing FEM calculation for only one case. All other cases can then be calculated by using analytical relations proposed here.

\section{List of symbols}

$T_{1}=$ Maximum temperature of the surface of the crystal

$T_{2}=$ Temperature of the copper water interface

$T_{3}=$ Mean temperature of out flowing water

$T_{f}=$ Temperature of in flowing water

$T_{I}=$ Temperature of silicon-copper interface for indirect cooling case

$\Delta T_{\max }=T_{1}-T_{f}$

$\Delta T_{i j}=T_{i}-T_{j}$

$h_{f}=$ Convection film coefficient

$P_{a b s}=$ Absorbed power for $1 \mathrm{mrad}$ horizontal fan

$P_{\max }=$ Maximum power density

$N=$ Number of milirad absorbed

$K=$ Thermal conductivity

$m=$ Mass of water flowing per second

$C_{v}=$ Specific heat of water

$D=$ Thickness of the crystal above the cooling channel 
$D_{\text {eff }}=$ Effective thickness of the crystal

$\theta=$ Bragg angle

$\sigma=$ Vertical divergence of the source

$A=$ Area of cross section of the cooling channel (figure 1 for details)

$\theta_{\max }=$ Maximum slope error

$H=$ Bump height

$V_{a}=$ Fluid flow velocity

$d=$ Equivalent diameter of the cooling channel

$v=$ Kinematic viscosity of water

$\alpha=$ Coefficient of thermal expansion

\section{References}

ANSYS-5.7 1998 Engineering analysis systems (Houston, PA: Swanson Analysis Systems Inc) 15342 Arthur J, Tompkins W H, Troxel Jr C, Contolini R J, Schmitt E, Bilderback D H, Henderson C, White J, Settersten T 1992 Rev. Sci. Instrum. 63: 433

Assoufid L, Lee W, Mills D M 1995 Rev. Sci. Instrum. 66: 2713

Berman L E, Hart H 1992 Rev. Sci. Instrum. 63: 437

Freund A K, Marot G, Kawata H, Joksch S, Ziegler E, Berman L E, Hastings J B 1992 Rev. Sci. Instrum. 63: 442

Jensen B N, Mancini D C, Nyholm R 1995 Rev. Sci. Instrum. 66: 2129

Knapp G S, Rogers C S, Beno M A, Wiley C L, Jennings G, Cowan P L 1995 Rev. Sci. Instrum. 66: 2139

Kuzay T M 1992 Rev. Sci. Instrum. 63: 468

Lenardi C 1993 Lecture notes, Second School on the Use of Synchrotron Radiation in Science and Technology (Unpublished)

Michaud F D 1986 Nucl. Instrum. Methods in Phys. Res. A246: 444

Mochizuki T, Zhang X, Sugiyama H, Zhao J, Ando M, Yoda Y 1995 Rev. Sci. Instrum. 66: 2167

Oversluizen T, Matsushita T, Ishikawa T, Stefan P M, Sharma S, Mikuni A 1989 Rev. Sci. Instrum. 60: 1493

Raja Rao A S 1998 Indus-2 project report Center for Advanced Technology, Indore

Rohsenow W M, Choi H Y 1961 Heat, Mass and Momentum Transfer (NJ: Prentice Hall Englewood Cliff), Chap 5-11: Chapman A J 1974 Heat Transfer (New York: Macmillan), Chap 5-13

Smither R K, Forster G A, Bilderback D H, Bedzyk M, Finkelstein K, Henderson C, White J, Berman L E, Stefan P, Oversluizen T 1989 Rev. Sci. Instrum. 60: 1486

Wang Z, Yun W, Kuzay T M, Knapp G, Rogers S 1995 Rev. Sci. Instrum. 66: 2267 